hep-th/0304051

FIT HE - 03-02

KYUSHU-HET 65

\title{
Dilaton coupled brane-world and field trapping
}

\author{
Kazuo Ghoroku ${ }^{\dagger}$, Motoi Tachibana ${ }^{\ddagger}$ and Nobuhiro Uekusa ${ }^{\S}$ \\ ${ }^{\dagger}$ Fukuoka Institute of Technology, Wajiro, Higashi-ku \\ Fukuoka 811-0295, Japan \\ $\ddagger$ Theoretical Physics Laboratory, RIKEN, 2-1 Hirosawa, \\ Wako, Saitama 351-0198, Japan

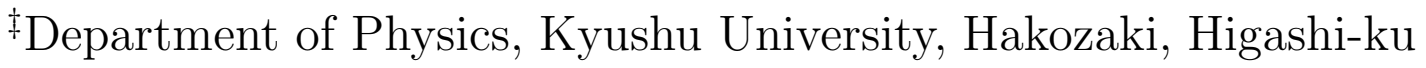 \\ Fukuoka 812-8581, Japan
}

\begin{abstract}
We address solutions of brane-world with cosmological constant $\lambda$ by introducing the dilaton in $5 \mathrm{~d}$ bulk, and we examine the localization of graviton, gauge bosons and dilaton. For those solutions, we find that both graviton and gauge bosons can be trapped for either sign, positive or negative, and wide range of $\lambda$ due to the non-trivial dilaton. While the dilaton can not be trapped on the brane.
\end{abstract}

${ }^{\dagger}$ gouroku@dontaku.fit.ac.jp

$\ddagger_{\text {motoi@postman.riken.go.jp }}$

§uekusa@higgs. phys. kyushu-u.ac.jp 


\section{Introduction}

The idea of the brane-world given in [1, 2] is strongly motivated by the recent development in the superstring/M theory [3, 4. In string theory however, the dilaton is necessarily included as a scalar partner of the graviton and plays many important dynamical roles. For example, the bulk configuration of the dilaton would give an insight for the running coupling constant of the boundary 4d Yang-Mills theory in an appropriate 10d background manifold. It would therefore be interesting to see the role of the dilaton in the brane-world and to examine whether the dilaton could live also in our $4 \mathrm{~d}$ world after the warped compactification and how it behaves. When it survives on the brane as a massless scalar partner of the graviton as in 10d, the equivalence principle, which will be observed in the free-fall experiments, would be remained as a problem to be resolved as in [5]. While it could appear as a $4 \mathrm{~d}$ massive scalar, its mass $\left(m_{\phi}\right)$ should be very small since it could not exceed the $4 \mathrm{~d}$ cosmological constant $\lambda$ in order to be trapped [23. Then we can estimate its magnitude as $m_{\phi} \sim \sqrt{\lambda}$, and the coupling to the light particles like photons could be estimated as $1 / M_{\mathrm{pl}}$. This implies a very long life time $\tau \sim M_{\mathrm{pl}}^{2} / m_{\phi}^{3}$ of the massive dilaton, and this fact gives a serious difficulty known as the moduli problem [6, 7, 8. In this case, we could however consider this massive particle as a candidate of the dark matter [9, 10.

It would be meaningful to study the problem of localization of the dilaton in order to approach to the above issues from the viewpoint of the brane-world. Although the localization of the graviton has been extensively studied [2, 11, 12, 18, 19, 20], much attention has not been given to scalar field 21, 22, 23], especially for the dilaton. However, it would be important to see the situation of the dilaton as well as the graviton in the context of the warped compactification within the superstring theory.

In addition to the dilaton and graviton, we also examine the localization of gauge bosons which are the important force of our $4 \mathrm{~d}$ world. Up to now, the localization of the graviton has been assured in a wide range of solutions except for $\mathrm{AdS}_{4}$ brane [11, 12. As for the gauge bosons, its localization has been examined in some models [13, 14, 15, 16, 17, 18, 24, 27. To trap gauge bosons, it was necessary to introduce positive $\lambda$ or the bulk-dilaton field. The latter case might be related to the massive vector model [24] by a special form of brane-vector coupling [28]. This point is an another reason to examine the brane-world with bulk-dilaton.

We study these issues in terms of a simple class of solutions for brane-world with non-trivial dilaton, which are essentially equivalent to the one given in [29]. However, the use and extension of these solutions are different. The dilaton $(\phi)$ is identified here with a scalar field which couples to the brane like $e^{\gamma \phi}$ and has the bulk potential of the exponential form. We concentrate on the localization of the fields mentioned above. Since the solutions given here have the same form of warp factor with the one, which are given before [19] for the case without the dilaton, then many problems of the localization would be easily understood.

Several new aspects caused by dilaton are given here. The localization of both the graviton and gauge bosons is realized for any brane of positive, negative and zero $\lambda$, i.e., $\mathrm{dS}_{4}, \mathrm{AdS}_{4}$ and Minkowski brane. When dilaton is absent, the gauge bosons are localized only for $\lambda>0, \mathrm{dS}_{4}$ brane [27, but this is not a necessary condition when we consider dilaton. The essential point needed for the localization of gauge bosons would 
be in the deformation of the warp factor from its exact AdS form. And the necessary deformation can be obtained either by the positive $\lambda$ or by the dilaton. In any case, the conformal symmetry is broken, so we could say that this breaking of the conformal symmetry would be inevitable for the localization of gauge bosons.

As for the graviton, it can be localized even if $\lambda<0$ when dilaton is included. This point is important since the general coordinate invariance is not necessarily broken for $\mathrm{AdS}_{4}$ brane in the case when dilaton is considered.

As for the localization of dilaton, we must solve the mixed equations with the scalar components of the metric. For our solutions, the master equation for these system is obtained in a simple form, and we find that any mode of the bulk dilaton can not be trapped on the brane.

In Section 2, we set our model with dilaton, and brane solutions are shown. We comment on the relation between these new solutions and the one given before without dilaton. In Section 3, the localization of graviton, gauge bosons and dilaton are examined. In the final section, summary and discussions are given.

\section{Dilaton coupled brane solution}

We begin with the following action with dilaton $(\phi)$,

$$
S_{g}=\int d^{4} x d y \sqrt{-g}\left\{\frac{1}{2 \kappa^{2}}(R-2 \Lambda)-\frac{1}{2}(\partial \phi)^{2}-V(\phi)\right\}-\tau \int d^{4} x \sqrt{-\operatorname{det} g_{\mu \nu}} e^{\gamma \phi},
$$

where the parameters are the five-dimensional gravitational constant $\kappa^{2}$, bulk cosmological constant $\Lambda$, brane tension $\tau$ and dilaton-brane coupling $\gamma$. The brane is set at $y=0$ by imposing $y \rightarrow-y$ symmetry on the above action. And the form of the potential $V$ is not specified at this stage. The equations of motion derived from the action (II) are given as

$$
\begin{aligned}
G_{M N}= & \kappa^{2}\left\{\partial_{M} \phi \partial_{N} \phi-g_{M N}\left(\frac{1}{2}(\partial \phi)^{2}+\frac{\Lambda}{\kappa^{2}}+V\right)-g_{\mu \nu} \delta_{M}^{\mu} \delta_{N}^{\nu} \tau e^{\gamma \phi} \delta(y)\right\}, \\
& \frac{1}{\sqrt{-g}} \partial_{M}\left\{\sqrt{-g} g^{M N} \partial_{N} \phi\right\}=\frac{\partial V}{\partial \phi}+\frac{\sqrt{-\operatorname{det} g_{\mu \nu}}}{\sqrt{-g}} \tau \gamma e^{\gamma \phi} \delta(y) .
\end{aligned}
$$

Here we solve these under the following ansatz for metric,

$$
d s^{2}=g_{M N} d x^{M} d x^{N}=A^{2}(y)\left(-d t^{2}+a^{2}(t) \gamma_{i j} d x^{i} d x^{j}\right)+d y^{2},
$$

where $\gamma_{i j}=\left(1+k \delta_{m n} x^{m} x^{n} / 4\right)^{-2} \delta_{i j}$. As long as we do not mention, $k=0$. While $a(t)$ is solved for each $k$, for example $a_{0}=e^{H_{0} t}, H_{0}=\sqrt{\lambda}$ for $k=0$. If we take as $\phi=\phi(y)$, Einstein equations (2) and dilaton equation (3) are written as

$$
\begin{gathered}
\frac{A^{\prime \prime}}{A}+\left(\frac{A^{\prime}}{A}\right)^{2}-\frac{\lambda}{A^{2}}=-\frac{\kappa^{2}}{3}\left(\frac{1}{2}\left(\phi^{\prime}\right)^{2}+\frac{\Lambda}{\kappa^{2}}+V(\phi)\right)-\frac{\kappa^{2} \tau}{3} e^{\gamma \phi} \delta(y) \\
\left(\frac{A^{\prime}}{A}\right)^{2}-\frac{\lambda}{A^{2}}=\frac{\kappa^{2}}{6}\left(\frac{1}{2}\left(\phi^{\prime}\right)^{2}-\frac{\Lambda}{\kappa^{2}}-V(\phi)\right) \\
\phi^{\prime \prime}+4 \phi^{\prime} \frac{A^{\prime}}{A}=\frac{\partial V}{\partial \phi}+\tau \gamma e^{\gamma \phi} \delta(y)
\end{gathered}
$$


where $^{\prime}=d / d y$. Let us assume the following form for $\phi$,

$$
\phi^{\prime}=\alpha A^{n} \operatorname{sgn}(\mathrm{y})
$$

Eqs.(6) and (17) are solved as

$$
\begin{aligned}
& \left(\frac{A^{\prime}}{A}\right)^{2}-\frac{\lambda}{A^{2}}=-\frac{\kappa^{2}}{6}\left(\frac{2 \alpha^{2}}{n} A^{2 n}+V_{0}\right)-\frac{\Lambda}{6}, \\
& V=\frac{n+4}{2 n}\left(\alpha A^{n}\right)^{2}+V_{0}, \quad 2 \alpha=\gamma \tau e^{\gamma \phi_{0}},
\end{aligned}
$$

where $V_{0}$ is a constant, and we take the boundary conditions $A(0)=1$ and $\phi(0)=\phi_{0}$. From eq.(9), we can see that the parameters $V_{0}$ and $\alpha$ can be absorbed into $\Lambda$ and $\lambda$ when $n=-1$. We can solve for other values of $n$, but we consider this simple solution in the following analysis since it is included in the previously obtained one for the case without dilaton by replacing the modified parameters. We should however notice here that this solution of $n=-1$ corresponds to the one which has already been found by P. Kanti et al.[29]. Another ansatz which could lead to the solutions similar to our previous ones are also possible, but they are a little bit complicated. So we concentrate here on the solution of $n=-1$ with the above ansatz (8).

Then the potential is given as

$$
V=-\frac{3}{2}\left(\frac{\alpha}{A}\right)^{2}+V_{0}
$$

and the equations (15) and (6) can be written as

$$
\begin{gathered}
\frac{A^{\prime \prime}}{A}+\left(\frac{A^{\prime}}{A}\right)^{2}-\frac{\tilde{\lambda}}{A^{2}}+\frac{\tilde{\Lambda}}{3}=-\frac{\kappa^{2} \tau}{3} e^{\gamma \phi_{0}} \delta(y) . \\
\left(\frac{A^{\prime}}{A}\right)^{2}+\frac{\tilde{\Lambda}}{6}=\frac{\tilde{\lambda}}{A^{2}} \\
\tilde{\Lambda}=\Lambda+\kappa^{2} V_{0}, \quad \tilde{\lambda}=\lambda+\frac{\kappa^{2} \alpha^{2}}{3} .
\end{gathered}
$$

As stated above, the equations (12) and (13) are the same form with the one obtained before [19] for the case where dilaton is suppressed. The dilaton modifies superficially the brane and bulk cosmological constants $(\lambda, \Lambda)$ to $(\tilde{\lambda}, \tilde{\Lambda})$ as shown above. So the solutions are obtained by replacing the parameters $(\lambda, \Lambda)$ in the previously given solutions by the new one, $(\tilde{\lambda}, \tilde{\Lambda})$. Then the various analyses can be performed in a parallel way. For example, the solutions $A(y)$ and $\phi(y)$ and the potential $V(\phi)$ are given as follows.

For $\tilde{\lambda}>0, \tilde{\Lambda}=0$,

$$
\begin{gathered}
A(y)=1-\sqrt{\tilde{\lambda}}|y| \equiv 1-\frac{|y|}{y_{H}}, \\
\phi(y)=-\frac{\alpha}{\sqrt{\tilde{\lambda}}} \ln (1-\sqrt{\tilde{\lambda}}|y|)+\phi_{0}, \quad V(\phi)=-\frac{3 \alpha^{2}}{2} e^{\frac{2 \sqrt{\tilde{\lambda}}}{\alpha}\left(\phi-\phi_{0}\right)}+V_{0} .
\end{gathered}
$$


For $\tilde{\lambda}>0, \tilde{\Lambda}<0$,

$$
\begin{gathered}
A(y)=\frac{\sqrt{\tilde{\lambda}}}{\mu} \sinh \left[\mu\left(y_{H}-|y|\right)\right], \\
\phi(y)=\frac{\alpha}{\sqrt{\tilde{\lambda}}} \ln \left(\frac{\operatorname{coth}\left[\mu\left(y_{H}-|y|\right) / 2\right]}{\operatorname{coth}\left(\mu y_{H} / 2\right)}\right)+\phi_{0}, \\
V(\phi)=-\frac{3 \alpha^{2}}{2} \frac{\mu^{2}}{\tilde{\lambda}} \sinh ^{2}\left\{\sqrt{\tilde{\lambda}}\left(\phi-\phi_{0}\right) / \alpha+\chi\right\}+V_{0}, \\
\mu=\sqrt{-\tilde{\Lambda} / 6}, \quad \sinh \left(\mu y_{H}\right)=\mu / \sqrt{\tilde{\lambda}}, \quad e^{\chi}=\operatorname{coth}\left(\mu y_{H} / 2\right) .
\end{gathered}
$$

For $\tilde{\lambda}>0, \tilde{\Lambda}>0$,

$$
\begin{gathered}
A(y)=\frac{\sqrt{\tilde{\lambda}}}{\mu_{d}} \sin \left[\mu_{d}\left(y_{H}-|y|\right)\right], \phi(y)=\frac{\alpha}{\sqrt{\tilde{\lambda}}} \ln \left(\frac{\cot \left[\mu_{d}\left(y_{H}-|y|\right) / 2\right]}{\cot \left(\mu_{d} y_{H} / 2\right)}\right)+\phi_{0}, \\
V(\phi)=-\frac{3 \alpha^{2}}{2} \frac{\mu_{d}^{2}}{\tilde{\lambda}} \cosh ^{2}\left\{\sqrt{\tilde{\lambda}}\left(\phi-\phi_{0}\right) / \alpha+\chi_{d}\right\}+V_{0}, \\
\mu_{d}=\sqrt{\tilde{\Lambda} / 6}, \quad \sin \left(\mu_{d} y_{H}\right)=\mu_{d} / \sqrt{\tilde{\lambda}}, \quad e^{\chi_{d}}=\cot \left(\mu_{d} y_{H} / 2\right) .
\end{gathered}
$$

For any above solution, there exists horizon whose position is represented by $y=y_{H}$ and which has curvature singularity due to the non-trivial dilaton. So we interpret the extra dimension is in a finite region, $0 \leq y \leq y_{H}$. Hereafter we set $\phi_{0}=0$ for the sake of brevity, or we interpret $\tau$ as $\tau e^{\gamma \phi_{0}}$ as long as we do not mention it especially. And we obtain from eq.(12) for the above solutions

$$
\tilde{\lambda}=\frac{\tilde{\Lambda}}{6}+\frac{\kappa^{4} \tau^{2}}{36} .
$$

This is the same form of the relation given before by $\Lambda$ and $\lambda$ in the case of the theory without dilaton.

\section{Localization}

Here we consider the localization of the bulk fields in terms of the linearized field equations for the graviton, dilaton and gauge bosons around the background configuration obtained above.

\subsection{Graviton}

Under the metric (4), it is convenient to take the gravitational fluctuation $h_{i j}$ as follows,

$$
d s^{2}=A(y)^{2}\left(-d t^{2}+a^{2}(t)\left[\gamma_{i j}\left(x^{i}\right)+h_{i j}\left(x^{\mu}, y\right)\right] d x^{i} d x^{j}\right)+d y^{2} .
$$

We are interested in the localization of the traceless transverse component, which represents the graviton on the brane. It is projected out by the conditions, $h_{i}^{i}=0$ and $\nabla_{i} h^{i j}=0$, where $\nabla_{i}$ denotes the covariant derivative with respect to the three-metric 
$\gamma_{i j}$ which is used to raise and lower the three-indices $i, j$. The transverse and traceless part is denoted by $h$ hereafter for simplicity. The linearized field equation for $h$ is obtained in the same form with the one given previously for the case without dilaton,

$$
\nabla_{5}^{2} h=0
$$

in terms of the five dimensional covariant derivative $\nabla_{5}^{2}=\nabla_{M} \nabla^{M}$. The above equation is given for $k=0$, and this is equivalent to the field equation of a five dimensional free massless scalar. For $k= \pm 1$, the term proportion to this appears, but it is not essential here and is abbreviated.

Then we arrive at the conclusion that the graviton can be localized on the brane if $\tilde{\lambda} \geq 0$. The new point is that the graviton can be trapped even if $\lambda<0$ differently from the case without dilaton. When the dialton is not considered, massive spin- 2 field is trapped on the brane of negative $\lambda$. Then the general coordinate invariance is broken for $\mathrm{AdS}_{4}$ brane from the viewpoint of the brane world scenario. But this is not always true as seen above.

For scalar fields, we can obtain similar results given for the case without dilaton by replacing the parameters $(\Lambda, \lambda)$ by $(\tilde{\Lambda}, \tilde{\lambda})$. The discussions are abbreviated here.

\subsection{Dilaton}

Here we discuss the dilaton according to its linearized equation around the background solution given here. The equation is obtained by denoting as $\phi=\bar{\phi}+\delta \phi$, where $\bar{\phi}$ represents the classical solution for $\phi$. The bulk "mass" term of the dilaton is obtained from the potential, $V(\bar{\phi}+\delta \phi)$ given above. Expanding it around the classical solutions, we get

$$
\begin{gathered}
V=\bar{V}+\frac{1}{2} m_{\phi}^{2}(\delta \phi)^{2}, \\
m_{\phi}^{2}=-\frac{6 \tilde{\lambda}}{A^{2}}+\frac{\tilde{\Lambda}}{2}
\end{gathered}
$$

where $\bar{V}=V(\bar{\phi})$. The explicit forms of $m_{\phi}^{2}$ for each solutions are written as

$$
\begin{array}{cc}
m_{\phi}^{2}=-6 \tilde{\lambda} e^{\frac{2 \sqrt{\lambda}}{\alpha} \bar{\phi}}, & \text { for } \tilde{\Lambda}=0, \tilde{\lambda}>0, \\
m_{\phi}^{2}=-3 \mu^{2} \cosh \left[2\left(\frac{\sqrt{\tilde{\lambda}}}{\alpha} \bar{\phi}+\chi\right)\right], & \text { for } \tilde{\Lambda}<0, \tilde{\lambda}>0, \\
m_{\phi}^{2}=-3 \mu_{d}^{2} \cosh \left[2\left(\frac{\sqrt{\tilde{\lambda}}}{\alpha} \bar{\phi}+\chi_{d}\right)\right], & \text { for } \tilde{\Lambda}>0, \tilde{\lambda}>0 .
\end{array}
$$

They are all not constant but the functions of $y$. In this sense, we can not see the mass of the dilaton in a normal form. On the other hand, we can see that they are all negative in all region of $y$. So the brane obtained here seems to be unstable for the dilaton fluctuation. However, we should notice the other mass term coming from the brane, and the total mass term is given as

$$
m_{\phi}^{2}=-\frac{6 \tilde{\lambda}}{A^{2}}+\frac{\tilde{\Lambda}}{2}+\tau \gamma^{2} \delta(y) .
$$


We can see a possibility to cure the instability by the third term. In order to make clear this point we must however solve the coupled equations with the scalar component of the metric and dilaton fluctuations.

In order to reduce the number of the scalar components of the metric, we move to the conformal coordinate by changing the fifth coordinate $y$ to $z$ in terms of the relation, $d y / d z=A(y)$. Then the metric is rewritten as

$$
d s^{2}=g_{M N} d x^{M} d x^{N}=A^{2}(z)\left(\gamma_{\mu \nu} d x^{\mu} d x^{\nu}+d z^{2}\right)
$$

where

$$
\gamma_{\mu \nu} d x^{\mu} d x^{\nu}=\left(-d t^{2}+a^{2}(t) \gamma_{i j} d x^{i} d x^{j}\right)
$$

In this coordinate, the scalar fluctuations of the metric are reduced to the following form in an appropriate gauge [26, 25],

$$
d s^{2}=A^{2}(z)\left[(1+2 \sigma) d z^{2}+(1+2 \psi) \gamma_{\mu \nu} d x^{\mu} d x^{\nu}\right] .
$$

After a calculation, we obtain the linearized equation for $\psi$,

$$
-\partial_{z}^{2} \psi-3 \mathcal{H} \partial_{z} \psi-\left(4 \partial_{z} \mathcal{H}+6 \lambda\right) \psi=\square_{4} \psi
$$

where $\mathcal{H}=\partial_{z} A / A$ and $\square_{4}$ denotes the four dimensional laplacian as given below. In deriving this equation, the constraints

$$
\sigma=-2 \psi, \quad \delta \phi \partial_{z} \bar{\phi}=-3\left(\partial_{z} \psi+2 \mathcal{H} \psi\right)
$$

and classical equations are used. In this process, the dilaton-brane coupling $\gamma$ is disappeared in (36) due to a characteristic property of our classical solution for the dilaton, the case of $n=-1$ in (8) . Then, $\psi$ is decomposed as follows in terms of the fourdimensional continuous mass eigenstates:

$$
\psi=\int d m \varphi_{m}\left(t, x^{i}\right) \Phi(m, y),
$$

where the $4 \mathrm{~d}$ mass $m$ is defined by

$$
-\square_{4} \varphi=\ddot{\varphi}_{m}+3 \frac{\dot{a}_{0}}{a_{0}} \dot{\varphi}_{m}+\frac{-\partial_{i}^{2}}{a_{0}^{2}} \varphi_{m}=-m^{2} \varphi_{m},
$$

and ${ }^{\cdot}=d / d t$. In order to see the localization, we do not need the solution of this equation, and we need only the explicit form of $\Phi(m, y)$. Its equation is rewritten into the form of one-dimensional Schrödinger-like equation with the rescaled $\Phi(m, y)$ as $\Phi(m, y)=A^{-3 / 2} u(z)$ and modified eigenvalue $\tilde{m}^{2}$,

$$
\left[-\partial_{z}^{2}+V(z)\right] u(z)=\tilde{m}^{2} u(z),
$$

where

$$
\tilde{m}^{2}=m^{2}+6 \lambda,
$$




$$
V(z)=\frac{9}{4}\left(A^{\prime}\right)^{2}-\frac{5}{2} A A^{\prime \prime}
$$

where we must notice ${ }^{\prime}=d / d y$. Since $A$ is singular at $y=0\left(\right.$ at $\left.z=z_{0}\right)$, then $u$ must satisfy the following boundary condition at $z=z_{0}$,

$$
u^{\prime}\left(z_{0}\right)=\frac{5 \kappa^{2} \tau}{12} u\left(z_{0}\right)
$$

And the eigenvalue $\tilde{m}^{2}$ of the bound state is given as the solution of this equation (43).

Without solving this equation, we can say that any state of $u(z)$ can not be trapped on the brane due to the positive $\delta$-function potential included in the second term of the above potential (42). We must notice that the potential becomes negative in some region for our background solutions, and this implies a tachyonic state could be trapped on the brane. But it is not a problem here since such a dangerous mode can not be trapped as stated above due to the property of the potential. So we can say that the brane considered here is stable for the dilaton fluctuation.

\subsection{Gauge field}

Here we consider a case where the dilaton couples to gauge fields with the following action

$$
\begin{aligned}
& S=S_{g}+S_{\text {gauge }} \\
& S_{\text {gauge }}=\int d^{4} x \int d y \sqrt{-g}\left(-\frac{1}{4} e^{4 \zeta \phi} g^{M N} g^{P Q} F_{M P} F_{N Q}\right),
\end{aligned}
$$

where $\zeta$ denotes the dilaton-gauge field coupling. When the dilaton is decoupled from the gauge fields, then $\zeta=0$ and the gauge fields can be trapped for $\tilde{\lambda}>0$ in this case. This is easily understood from the previous analysis [27] given for the case without dilaton. In fact, the warp factor and the equation of the gauge-boson fluctuation have the same form with the one given in [27] if $(\tilde{\lambda}, \tilde{\Lambda})$ were replaced by $(\lambda, \Lambda)$.

For $\zeta \neq 0$, we expand the fields in terms of the four-dimensional mass eigenstates, $A_{M}=\int d m a_{M}\left(t, x^{i}, m\right) \phi_{M}(y, m)$, and the equation of motion for the spatial transverse component $A_{i}^{T}$ is derived as

$$
\begin{array}{r}
\left(\partial_{y}^{2}+\left\{2 \frac{A^{\prime}}{A}+4 \zeta \phi^{\prime}\right\} \partial_{y}+\frac{m^{2}}{A^{2}}\right) \phi_{i}^{T}=0 \\
\left(-\partial_{t}^{2}-\frac{\dot{a}_{0}}{a_{0}} \partial_{t}+\frac{\partial_{j}^{2}}{a_{0}^{2}}\right) a_{i}^{T}=m^{2} a_{i}^{T} .
\end{array}
$$

By introducing $u(z)$ and $z$ defined as $\phi_{i}^{T}=A^{-1 / 2} u(z)$ and $\partial z / \partial y= \pm A^{-1}$, Eq.(46) can be written as

$$
\left[-\partial_{z}^{2}-4 \xi \partial_{z}+V(z)\right] u(z)=m^{2} u(z),
$$

where $\xi=\zeta \alpha$ and the potential $V(z)$ is given as

$$
V(z)=\frac{1}{4}\left(A^{\prime}\right)^{2}+\frac{1}{2} A A^{\prime \prime}+2 \xi A^{\prime} .
$$


It is difficult to get an analytic solution of the equation (48) for non-zero $m$ except for some special cases, which are given in two examples below. Before showing them, we show that the zero mode is trapped on the brane for all solutions given above. In other words, the gauge bosons can be trapped for those solutions when the dilaton couples to the gauge bosons. It is proved as follows.

For $\zeta=0$, the eigenvalue equation is written as

$$
\begin{gathered}
{\left[-\partial_{z}^{2}+V_{0}(z)\right] u(z)=m^{2} u(z),} \\
V_{0}(z)=\frac{1}{4}\left(A^{\prime}\right)^{2}+\frac{1}{2} A A^{\prime \prime},
\end{gathered}
$$

For the zero-mode $u_{0}(z)(m=0)$, Eq.(150) is written as,

$$
\left[-\partial_{z}^{2}+V_{0}(z)\right] u_{0}(z)=0
$$

and its normalizable solution is obtained as

$$
u_{0}(z)=c_{0} X^{1 / 4}
$$

where $c_{0}$ is a constant. And $X=\sinh ^{-2}(\sqrt{\tilde{\lambda}} z)\left(X=\cosh ^{-2}(\sqrt{\tilde{\lambda}} z)\right)$ for $\tilde{\lambda}>0$ and $\tilde{\Lambda}<0(\tilde{\Lambda}>0)$.

It is easily seen that the above solution $u_{0}(z)$ also satisfies the zero-eigenvalue equation of $\zeta \neq 0$,

$$
\left[-\partial_{z}^{2}-4 \xi \partial_{z}+V(z)\right] u_{0}(z)=0
$$

where $\xi=\zeta \alpha$ and the potential $V(z)$ is given as

$$
V(z)=V_{0}(z)+2 \xi A^{\prime}
$$

The proof of (54) is shown as follows. By substituting the solution (53) into (54), we find

$$
\left[-\partial_{z}^{2}-4 \xi \partial_{z}+V(z)\right] u_{0}(z)=2 \xi\left(-2 \partial_{z}+A^{\prime}\right) u_{0}(z)
$$

and the right hand side vanishes when the explicit forms of $u_{0}(z)$ and $A^{\prime}(z)$ for each solution given above are used. Then the zero-mode solutions are not affected by the dilaton coupling.

On the other hand, the normalizability condition is modified by the dilaton coupling. When dilaton couples to the gauge bosons, the condition of normalizability of zero mode is given as

$$
\int_{z_{0}}^{\infty} d z e^{4 \xi z} u_{0}(z)^{2}<\infty
$$

From this and the explicit form of $u_{0}(z)$, we find for both solutions the following common condition,

$$
4 \xi<\sqrt{\tilde{\lambda}}
$$

As a result, we can say that the gauge bosons can be trapped for the case of $\zeta \neq 0$ whenever the constraint (58) is satisfied. In the followings, we can see this relation through the explicit solutions for the soluble cases for general $\mathrm{m}$. 
As a first example, we consider the case of $\tilde{\lambda}>0$ and $\tilde{\Lambda}=0$. This is given as a limit of $\tilde{\Lambda} \rightarrow 0$ from either solution of $\tilde{\Lambda}>0$ or $\tilde{\Lambda}<0$. In this case, we can solve for $u(z)$ of non-zero $m$, but the setting of $\tilde{\Lambda}=0$ would not be realistic when we respect the Newton's law to be observed in our world. Although the graviton is trapped on the brane, its KK modes would be overwhelming [31]. So the analysis of this case is performed from the theoretical interest.

As above the same form of equation (48) and (49) are obtained. And the explicit form of the potential is given as

$$
V(z)=\frac{\sqrt{\tilde{\lambda}}}{4}(\sqrt{\tilde{\lambda}}-8 \xi)-\sqrt{\tilde{\lambda}} \delta\left(z-z_{0}\right) .
$$

Then $u(z)$ is solved as,

$$
u(z)=c_{1} e^{k_{+} z}+c_{2} e^{k_{-} z}, \quad k_{ \pm}=-2 \xi \pm \sqrt{(2 \xi-\sqrt{\tilde{\lambda}} / 2)^{2}-m^{2}}
$$

where $c_{1}$ and $c_{2}$ are constants of integration. The normalizable solution is obtained by choosing $k_{-}$and the boundary condition, (67), can be written as

$$
-2 \xi+\sqrt{\tilde{\lambda}} / 2=\sqrt{(2 \xi-\sqrt{\tilde{\lambda}} / 2)^{2}-m^{2}} .
$$

Then we obtain $m=0$ and $\xi<\sqrt{\tilde{\lambda}} / 4$, which is nothing but the one given above, (58). This indicates that the result given above for the zero-mode is satisfied also in the limit of $\tilde{\Lambda} \rightarrow 0$.

As a second soluble example, we consider another limit, $\tilde{\lambda}=0$. However, $\lambda$ is zero or negative in this limit as seen from the relation $\tilde{\lambda}=\lambda+\frac{\kappa^{2} \alpha^{2}}{3}$ (see (14)), so the brane is not a realistic also in this case when we respect the present observation of small but positive $\lambda$. However it is meaningful from a theoretical viewpoint to study this case.

In this case, the warp factor $A(y)$ takes the simple Randall-Sundrum form, and the potential is given as

$$
V(z)=\frac{3}{4 z^{2}}-\frac{2 \xi}{z}-\mu \delta\left(|z|-z_{0}\right)
$$

Then $u(z)$ is solved as,

$$
u(z)=e^{-2 z(\xi+d / 2)} z^{3 / 2}\left\{c_{1}{ }_{1} F_{1}\left(b_{1}, b_{2} ; 2 z d\right)+c_{2} U\left(b_{1}, b_{2} ; 2 z d\right)\right\},
$$

where $c_{1}$ and $c_{2}$ are constants of integration and

$$
d=\sqrt{4 \xi^{2}-m^{2}}, \quad b_{1}=\frac{3}{2}-\frac{\xi}{d}, \quad b_{2}=3
$$

Here ${ }_{1} F_{1}\left(b_{1}, b_{2} ; X\right)$ denotes the Kummer's hypergeometric function, and the confluent hypergeometric function denoted by $U\left(b_{1}, b_{2} ; X\right)$ is another independent solution of the same differential equation. It follows from this solution that $u(z)$ oscillates with $z$ when $m^{2}>4 \xi^{2}$, where the continuum KK modes appear.

Here we concentrate on the bound state which is restricted to the region of $m^{2}<4 \xi^{2}$. From the normalization condition, which is obtained by replacing $u_{0}(z)$ in (57) by $u(z)$,

$$
\int e^{4 \xi z} u^{2}(z) d z<\infty
$$


the solution for the localized state is obtained by setting $c_{1}=0$ since

$$
e^{2 \xi z} e^{-2 z(\xi+d / 2)} z^{3 / 2}{ }_{1} F_{1}\left(b_{1}, b_{2} ; 2 z d\right) \rightarrow e^{2|\xi| z} / \sqrt{z}
$$

for $z \rightarrow \infty$. Then $u(z)$ is written as,

$$
u(z)=c_{2} e^{-2 z(\xi+d / 2)} z^{3 / 2} U\left(b_{1}, b_{2} ; 2 z d\right) .
$$

At the next step, we consider the boundary condition at $z=z_{0}$ (or $y=0$ ), which is given as follows by taking into account of the $\delta$-function potential in (62),

$$
\frac{d u\left(z_{0}\right)}{d z}=-\frac{1}{2}\left(\frac{\kappa^{2} \tau}{6}\right) u\left(z_{0}\right)
$$

This condition can be written as

$$
\frac{1}{2}+\frac{\mu-\xi}{d}=\frac{U\left(b_{1}, b_{2}+1 ; 2 d / \mu\right)}{U\left(b_{1}, b_{2} ; 2 d / \mu\right)},
$$

where we used $z_{0}=1 / \mu$. This equation provides the value of $m$ when $\xi$ and $\mu$ are given. Our interest is in the trapped gauge bosons, so we see this equation for $m=0$. For $\xi>0$, the above equation is written as

$$
\frac{2}{x}=\frac{U(1,4 ; x)}{U(1,3 ; x)}
$$

where $x=4|\xi| / \mu$. While the right hand side is obtained as $\S$

$$
\frac{U(1,4 ; x)}{U(1,3 ; x)}=\frac{2}{x}+\frac{x}{1+x}
$$

and we can see (69) is not satisfied for $x>0$. From this we can say that there is no solution of $m=0$ for $\xi>0$.

For $\xi<0$, equation (68) is written as

$$
1+\frac{2}{x}=\frac{U(2,4 ; x)}{U(2,3 ; x)}
$$

This equation is the identity, so we find always the solution $m=0$ for $\xi<0$. As a result, we can say that the gauge bosons can be trapped in the region, $\xi<0$, for $\tilde{\lambda}=0$. This result is again consistent with the relation $4 \xi<\sqrt{\tilde{\lambda}}$, (58). In order to see clearly the above result, the numerical estimation of the value of $m$ as the solutions of (67) is shown in the Fig 1 as a function of $\xi$ for fixed $\mu, \mu=1$.

For the case of $\tilde{\lambda}<0$, the graviton can not be trapped as stated above, then this brane is also unrealistic. While it would be worthwhile from the theoretical viewpoint to study this case, but we like to discuss in the future.

Finally at this sub-section, we comment on another interesting point stated in the introduction for the case of nonzero $\zeta$. The dilaton-coupled action (44) could be related

\footnotetext{
${ }^{\S}$ The relations (70) and (71) are easily obtained from the identities, $U(a, a+1 ; x)=x^{-a}$ and $U^{\prime}(a, c ; x)=U(a, c ; x)-U(a, c+1 ; x)$
} 


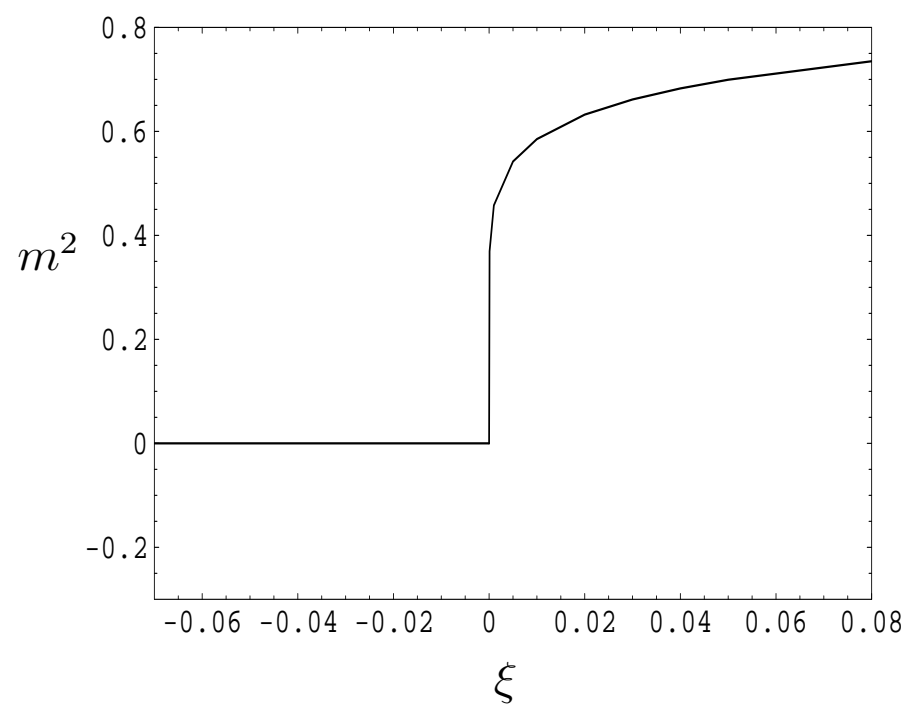

Fig. 1: The value $m^{2}$ (vertical-axis) of the solution of equation (67) versus $\xi$ (horizontalaxis) for $\mu=1$.

to the action described in terms of massive vector field. According to [28, where the case of zero effective cosmological constant $\lambda$ is considered, we define the massive vector field as $\tilde{A}_{M} \equiv \epsilon A_{M}$ and $\epsilon \equiv e^{2 \zeta \phi}$. Then the mass-like term for the component $\tilde{A}_{\mu}$ is given by

$$
-\left(\frac{A^{\prime}}{A} \frac{\epsilon^{\prime}}{\epsilon}+\frac{1}{2}\left(\frac{\epsilon^{\prime}}{\epsilon}\right)^{\prime}+\frac{1}{2}\left(\frac{\epsilon^{\prime}}{\epsilon}\right)^{2}\right) \sqrt{-g} g^{\mu \nu} \tilde{A}_{\mu} \tilde{A}_{\nu},
$$

where the delta function term is also included. For the brane solutions given above, the "mass" is dependent on $y$ in general, then it can not be identified as the standard mass. This would imply that we need special brane solution to rewrite the theory to the massive gauge theory with a special coupling to the brane. On this point, we will discuss in a separate paper.

\section{Summary and Discussions}

We have examined the solutions of brane-world including the dilaton and investigated the localization of the bulk fields. Due to a specific ansatz, the solutions are obtained in a way where only the brane and bulk cosmological constant $(\lambda, \Lambda)$ are modified to $(\tilde{\lambda}, \tilde{\Lambda})$ without changing the equations of motion compared to the case without dilaton field. The new point is the appearance of a dynamical bulk scalar, the dilaton.

The solutions are classified by these new parameters $(\tilde{\lambda}, \tilde{\Lambda})$. For $\tilde{\lambda}>0$, the graviton and the gauge bosons are trapped as shown previously. The new feature is seen from $\tilde{\lambda}=\lambda+\kappa^{2} \alpha^{2} / 3$. When dilaton is decoupled $(\alpha=0)$, the graviton is trapped only for $\lambda \geq 0$, but it is trapped even if $\lambda<0$ when the dilaton couples and $\tilde{\lambda}>0$ were satisfied. This is always possible since $\kappa^{2} \alpha^{2} / 3>0$. This point is important in the sense that we can see the graviton also in $\mathrm{AdS}_{4}$ brane. The breaking of the general coordinate invariance is expected in $\mathrm{AdS}_{4}$ brane, but this is evaded by including the 
dilaton in such a way to satisfy $\tilde{\lambda}>0$. We should however notice that this breaking would be seen in $\mathrm{AdS}_{4}$ brane when $\lambda$ is very small and we arrive at $\tilde{\lambda}<0$.

As for the gauge bosons, the new analyses are given here by including the coupling $(\zeta)$ of the dilaton to gauge bosons. We find that the gauge bosons are trapped for

small enough coupling $4 \xi(=4 \zeta \alpha)<\sqrt{\tilde{\lambda}}$ (see (58) ). This condition is further assured by studying two concrete examples, where solutions for any $4 \mathrm{~d}$ mass-state could be obtained.

When we consider the smallness of $\lambda$ and $\tilde{\lambda}=\lambda+(\kappa \alpha)^{2} / 3$, the above condition is satisfied if $\zeta$ is the same order of the 5 d gravitational coupling $\kappa$ or smaller than it. As a limit of this constraint, we can consider the case of $\zeta=0$, the decoupling limit. Our results given here are consistent with the one given in the previous analysis for $\zeta=0$ where the trapping of the gauge bosons is observed for small $\lambda$. One more point to be noticed is the relation between the dilaton coupled gauge theory and the massive vector theory. We will address on this point in near future.

Finally we comment on the localization of the dilaton which should be considered as an important dynamical field as well as graviton from the string theory viewpoint. At first glance, there seems a possible tachyonic bound state of the dilaton fluctuations since its bulk potential is negative. The trapping problem of this field however should be solved as a mixed system with scalar components of the metric fluctuations. We could then find a simple master equation for this system and that there is no trapping of any mode of dilaton fluctuations and also for the scalar components of the metric. As a result, we obtain a stable brane model with a dilaton. The massless dilaton is usually expected as in the 10d superstring theory, but our analysis implies that the dilaton is absent in our $4 \mathrm{~d}$ universe after a compactification. From the viewpoint of superstring theory, this seems to be a difficulty at a glance, but it could give a possible resolution for the moduli problem and the equivalence principle when the dilaton is rejected to exist in our $4 \mathrm{~d}$ world.

The bulk dilaton can be related to the problem of the AdS/CFT correspondence. When the dilaton is trivial or absent, the bulk is $\mathrm{AdS}_{5}$. And it has been observed that the propagator of the graviton on the brane seems to receive quantum corrections from CFT living on the same brane [32, 33]. This correction is seen through the Newton potential between two massive objects on the brane, and the same correction is obtained from the $5 \mathrm{~d}$ propagator of the graviton in the AdS bulk. This is known as AdS/CFT correspondence which is consistent with the conformal invariance. For the case of the non-trivial dilaton, we expect a non-conformal field theory on the boundary. So it would be an interesting problem to study this problem in our model with non-trivial dilaton configurations. A report on these points would be given in a future paper.

\section{Acknowledgments}

This work has been supported in part by the Grants-in-Aid for Scientific Research (13135223) of the Ministry of Education, Science, Sports, and Culture of Japan. M.T. was supported by Special Postdoctral Research Program in RIKEN. 


\section{References}

[1] L. Randall and R. Sundrum, Phys. Rev. Lett. 83, 3370 (1999),(hep-ph/9905221).

[2] L. Randall and R. Sundrum, Phys. Rev. Lett. 83, 4690 (1999),(hep-th/9906064).

[3] P. Horava and E. Witten, Nucl. Phys. B 460, 506 (1996) hep-th/9510209.

[4] J. M. Maldacena, Adv. Theor. Math. Phys. 2, 231 (1998) hep-th/9711200.

S. S. Gubser, I. R. Klebanov and A. M. Polyakov, Phys. Lett. B 428, 105 (1998) hep-th/9802109.

E. Witten, Adv. Theor. Math. Phys. 2, 253 (1998) hep-th/9802150.

[5] T. Damour, F. Piazza and G. Veneziano, Phys. Rev. Lett. 89, 081061 (2002), (gr-qc/0204094).

[6] T. Banks, D. Kaplan and A. Nelson, Phys. Rev. D 49, 779 (1994).

[7] L. Randall and S. Thomas, Nucl. Phys. B449, (1995) 229, hep-ph/9407248.

[8] T. Damour and A. Vilenkin, Phys. Rev. D53, 2981 (1996), hep-th/9503149.

[9] B. de Carlos, J.A. Casas, F. Quevedo and E. Roulet, Phys. Lett. B 318 (1993) 447.

[10] R. Dick, (hep-th/9609190).

[11] A. Karch and L. Randall, JHEP 0105 (2001) 008, (hep-th/0011156).

[12] A. Miemiec, Fortsch. Phys. 49 (2001) 747, (hep-th/0011160).

[13] G. Dvali and M. Shifman, Phys. Lett. B396 (1997) 64 [Erratum-ibid. B407 (1997) 452], (hep-th/9612128).

[14] G. Dvali, G. Gavadadze and M. Shifman, Phys. Lett. B497 (2001) 271, (hep-th/0010071).

[15] P. Dimopoulos, K. Farakos, A. Kehagias and G. Koutsoumbas, Nucl. Phys. B617 (2001) 237, hep-th/0007079.

[16] M.J. Duff, J.T. Liu, and W.A. Sabra, Nucl. Phys. B605 (2001) 234, hep-th/0009212.

[17] I. Oda, hep-th/0103052.

[18] A. Kehagias and K. Tamvakis, Phys. Lett. B504 (2001) 38, (hep-ph /0010112).

[19] I. Brevik, K. Ghoroku , S. D. Odintsov and M. Yahiro, Physical Review D 66 (2002) 064016-1-9.

[20] B. Bajc and G. Gabadadze, Phys. Lett. B474 (2000) 282, (hep-th/9912232). M. Ito, (hep-th/0204113). P. Singh and N. Dadhich, (hep-th/0208080). 
[21] S. Nojiri, O. Oberegon, S. Odintsov and S. Ogushi, Review D 62 (2000) 064017 , (hep-th/0003148).

[22] K. Ghoroku and A. Nakamura, Phys. Rev. D64 (2001) 084028, (hep-th/0103071).

[23] D. Langlois and M. Sasaki, (hep-th/0302069). K. Ghoroku, and M. Yahiro, (hep-th/0211112).

[24] K. Ghoroku and A. Nakamura, Physical Review D65 (2002) 084017-1-6.

[25] M. Giovannini, Physical Review D64 (2001) 064023.

[26] S. Kobayashi, K. Koyama and J. Soda, Physical Review D65 (2002) 064014-1-9.

[27] K. Ghoroku and N. Uekusa, hep-th/0212102

[28] M. Tachibana, hep-th/0108164.

[29] P. Kanti, S. Lee and K.A. Olive, Phys. Rev. D66 (2002) 125008, (hep-ph/0209036).

[30] M. Duff and P. van Nieuwenhuizen, Phys. Lett. B 94, 179 (1980).

[31] K. Ghoroku, A. Nakamura and M. Yahiro, hep-th/0303068

[32] M.J. Duff and J. T. Liu, Phys. Rev. Lett. 85 (2000) 2052, (hep-th/0003237).

[33] S.B. Giddings, E. Katz and L. Randall, JHEP 03 (2000) 023, (hep-th/0002091). 